\title{
Heparanase upregulation by colonic epithelium in inflammatory bowel disease
}

\author{
Matti Waterman¹, Ofer Ben-Izhak², Rami Eliakim¹, Gabriel Groisman³, Israel Vlodavsky ${ }^{4}$ \\ and Neta Ilan ${ }^{4}$ \\ ${ }^{1}$ Department of Gastroenterology, Rambam Health Care Campus, Haifa, Israel; ${ }^{2}$ Department of Pathology, \\ Rambam Health Care Campus, Haifa, Israel; ${ }^{3}$ Department of Pathology, Hillel-Yaffe Medical Center, \\ Hadera, Israel and ${ }^{4}$ Bruce Rappaport Faculty of Medicine, Cancer and Vascular Biology Research Center, \\ Technion-Israel Institute of Technology, Haifa, Israel
}

\begin{abstract}
Heparanase is an endo- $\beta$-D-glucuronidase capable of cleaving heparan sulfate (HS) side chains at a limited number of sites, yielding HS fragments of still appreciable size $(\sim 5-7 \mathrm{kDa})$. Heparanase activity has long been detected in a number of cell types and tissues. Importantly, heparanase activity correlated with the metastatic potential of tumor-derived cells, attributed to enhanced cell dissemination as a consequence of HS cleavage and remodeling of the extracellular matrix barrier. Similarly, heparanase activity was implicated in neovascularization, inflammation and autoimmunity, involving migration of vascular endothelial cells and activated cells of the immune system. The involvement of heparanase in inflammatory processes of the gastrointestinal tract has not been examined. Here, we utilized immunohistochemical analysis to investigate heparanase expression in acute and chronic inflammatory conditions. Heparanase expression was not detected in specimens derived from normal colon tissue. In contrast, strong heparanase staining was observed in Crohn's disease and ulcerative colitis, but not in infectious colitis. Interestingly, heparanase staining was primarily observed in epithelial rather than immune cells. Importantly, un-fractionated as well as low molecular weight heparin (enoxaparin), which exhibit a strong inhibitory activity towards heparanase, have proven efficacious in ulcerative colitis and Crohn's disease patients, suggesting that heparanase is actively involved in these pathologies and thus may be considered as a target for the development of anti-inflammatory therapies. Modern Pathology (2007) 20, 8-14. doi:10.1038/modpathol.3800710; published online 13 October 2006
\end{abstract}

Keywords: heparanase; immunostaining; inflammatory bowel disease; epithelium

The mammalian endoglycosidase heparanase is the predominant enzyme that degrades heparan sulfate (HS) side chains of heparan sulfate proteoglycans (HSPG), the dominant proteoglycan in the extracellular matrix (ECM) and cell surfaces. ${ }^{1-3}$ HSPG consist of a protein core to which HS side chains are covalently attached. These complex macromolecules are highly abundant in the ECM and are thought to play an important structural role, contributing to ECM integrity and insolubility. ${ }^{4,5}$ Traditionally, heparanase activity was well correlated with the metastatic potential of tumor-derived cells, attributed to enhanced cell dissemination as a consequence of HS cleavage and remodeling of the

Correspondence: Professor I Vlodavsky, PhD, Bruce Rappaport Faculty of Medicine, Cancer and Vascular Biology Research Center, Technion-Israel Institute of Technology, Technion, PO Box 9649, Haifa 31096, Israel.

E-mail: Vlodavsk@cc.huji.ac.il

Received 14 June 2006; revised and accepted 28 August 2006; published online 13 October 2006
ECM barrier. ${ }^{6-9}$ A proof-of-concept of this notion has recently been established by using a specific antiheparanase ribosyme and siRNA methodology, ${ }^{10}$ clearly implicating heparanase activity as a critical requisite for metastatic spread. Similarly, heparanase was implicated in cell dissemination associated with inflammation and autoimmunity. ${ }^{11-13}$ Furthermore, heparanase activity can liberate a variety of HSPG-bound biological mediators, including cytokines and chemokines such as interferon (IFN)- $\gamma$, MIP-1 $\beta$, RANTES and interleukins, thus contributing to the regulation of inflammation and other immune responses. ${ }^{14}$

Heparanase expression by the gastrointestinal tract has been documented by employing immunostaining, RT-PCR and heparanase activity analyses. ${ }^{15-17}$ In these studies, heparanase was not detected in normal looking mucosa, whereas high levels of expression were detected in primary and metastatic colon carcinoma. ${ }^{15-17}$ The involvement of heparanase in inflammatory processes typical for the gastrointestinal tract has not been so far 
examined. Crohn's disease and ulcerative colitis are chronic inflammatory conditions of unknown origin. Current data suggest that the combination of genetic, immunologic and environmental factors lead to chronic inflammatory conditions. ${ }^{18}$ Here, we utilized immunohistochemistry to investigate heparanase expression in acute and chronic inflammatory conditions. Heparanase expression was not detected in specimens derived from normal colon tissue, whereas strong heparanase staining was observed in Crohn's disease and ulcerative colitis, but not infectious colitis. Heparanase staining was primarily observed in epithelial rather than immune cells. These results indicate that heparanase levels are elevated under chronic inflammatory conditions, and that the colonic epithelium is the major source for heparanase in chronic, but not acute inflammatory disorders.

\section{Materials and methods}

\section{Experimental Design}

The study included archived formalin-fixed, paraffin-embedded colon sections obtained from ulcerative colitis $(n=17)$, Crohn's disease $(n=17)$ and infectious colitis patients $(n=8)$ who underwent surgical colonic resection or colonoscopy at the Rambam Health Care Campus, Haifa, Israel. Sociodemographic and clinical profile (age at diagnosis, gender, disease duration before surgery, type of resection, disease extent and behavior) were collected from medical records. Crohn's disease and ulcerative colitis patients were diagnosed by expert gastroenterologists reviewing endoscopic, pathology and radiology reports. The disease extent and behavior were determined according to Gasche et $a .^{19}$ Briefly, Crohn's disease patients were considered to have penetrating disease if intraabdominal, peri-anal fistulas, inflammatory masses and/or abscesses were evident at any time in the course of disease, excluding postoperative intraabdominal complications. Stricturing disease was defined as the occurrence of constant luminal narrowing with pre-stenotic dilatation or obstructive signs/symptoms without presence of penetrating disease. By exclusion, all other Crohn's disease patients were considered to have an inflammatory disease. Crohn's disease location was classified as terminal ileal, colonic, ileocolonic and upper-gastrointestinal (disease limited proximal to the ileum). Ulcerative colitis location was classified as proctitis, left-sided colitis or pancolitis.

Microbiological laboratory data of stool cultures from infectious colitis patients were reviewed. Positive cultures for Salmonella, Shigella, Campylobacter, parasites or Clostridium difficile toxin, were retrieved.

Normal colonic samples were obtained from 17 patients who underwent surgical colon resection for colorectal carcinoma. Formalin-fixed, paraffin- embedded sections of normal-looking mucosa distant from the tumor margins were analyzed.

The study protocol was approved by the Rambam Health Care Institutional Review Board.

\section{Evaluation of Inflammation Activity}

Tissue specimens were stained with hematoxyllin and eosin and classified by an expert pathologist as normal mucosa, mild, moderate or severe colitis, according to the proportion of sample crypt crosssections containing intraluminal or intraepithelial neutrophils, that is, crypt abscesses or cryptitis. For each sample, an activity score was determined by an expert pathologist ranking $0-3$ ( 0 , no activity; 1 , mild activity ( $<50 \%$ of crypts); 2 , moderate activity (at least $50 \%$ of crypts, but no erosion/ulceration); 3 , severe activity (erosion/ulceration present)).

\section{Heparanase Immunostaining}

Staining of formalin-fixed, paraffin-embedded $5 \mu \mathrm{m}$ sections for heparanase was performed essentially as described. ${ }^{17,20}$ Briefly, slides were deparaffinized, rehydrated and endogenous peroxidase activity was quenched $(30 \mathrm{~min})$ by $3 \%$ hydrogen peroxide in methanol. Slides were then subjected to antigen retrieval by boiling ( $20 \mathrm{~min}$ ) in $10 \mathrm{mM}$ citrate buffer, $\mathrm{pH}$ 6. Slides were incubated with $10 \%$ normal goat serum in phosphate-buffered saline (PBS) for $60 \mathrm{~min}$ to block nonspecific binding and incubated $(20 \mathrm{~h}$, $4^{\circ} \mathrm{C}$ ) with anti-heparanase 733 or 810 antibodies, diluted 1:400 and 1:100, respectively in blocking solution. Antibody 733 was raised in rabbits against a 15 amino-acid peptide (KKFKNSTYSRSSVDC) that maps at the N-terminus of the $50 \mathrm{kDa}$ heparanase subunit, and preferentially recognizes the $50 \mathrm{kDa}$ heparanase subunit vs the $65 \mathrm{kDa}$ latent pro-enzyme (Figure 1b). ${ }^{20}$ Antibody 810 was raised in rabbits against a 13 amino-acid peptide (GTKTDFLIFDPKK) located at the C-terminus of the $8 \mathrm{kDa}$ heparanase subunit and preferentially recognizes the $8 \mathrm{kDa}$ heparanase subunit $v s$ the $65 \mathrm{kDa}$ latent enzyme (Figure 1b). ${ }^{21}$ Slides were extensively washed with PBS containing $0.01 \%$ Triton X-100 and incubated with a secondary reagent (Envision kit) according to the manufacturer's (Dako, Glostrup, Denmark) instructions. Following additional washes, color was developed with the AEC reagent (Dako), sections were counterstained with hematoxylin and mounted, as described. Immunostained specimens were examined by senior pathologists, blind to clinical data of the patients and were scored according to the intensity of staining ( 0 , no staining; 1 , weak; 2 , moderate; 3 , strong staining). Specimens that were similarly stained with pre-immune serum, or applying the above procedure but lacking the primary antibody, yielded no detectable staining. 


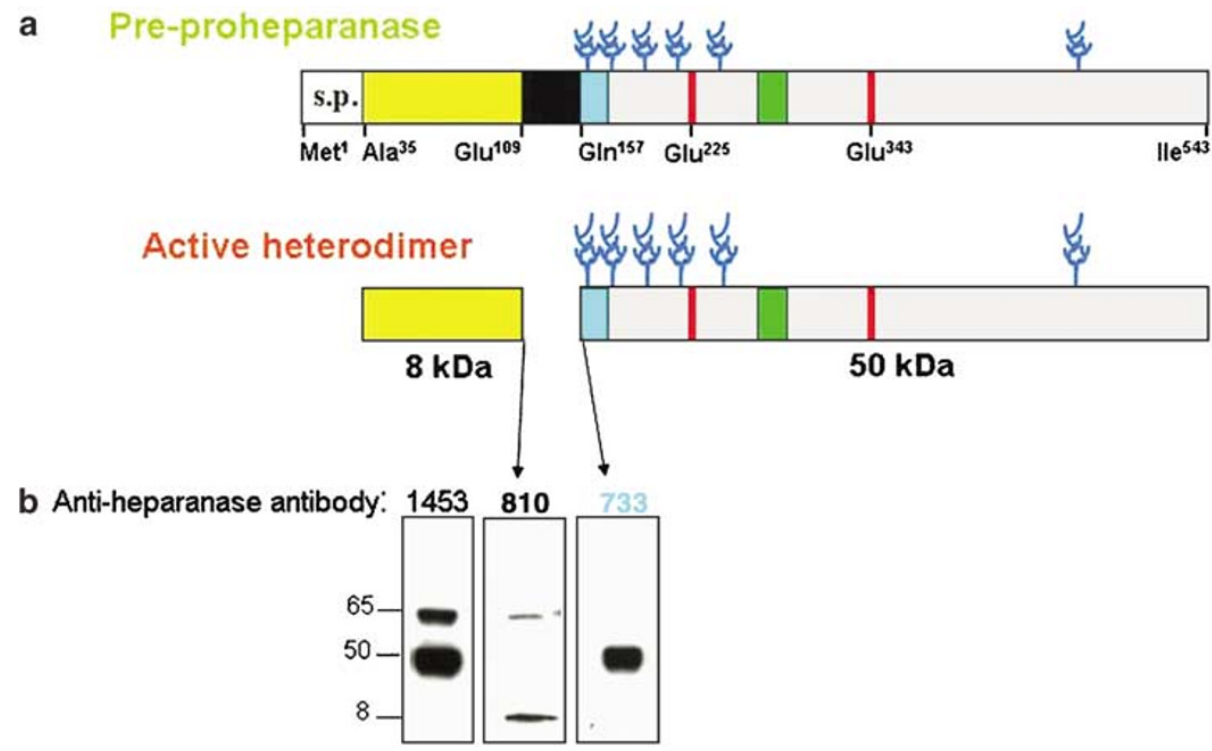

Figure 1 Heparanase primary structure, critical amino acids and antibody specificity. (a) Primary structure. Pre-proheparanase harbors a signal peptide (SP, Met $^{1}-\mathrm{Ala}^{35}$ ) which is removed upon entering the ER. The protein is then subjected to glycosylation at six N-

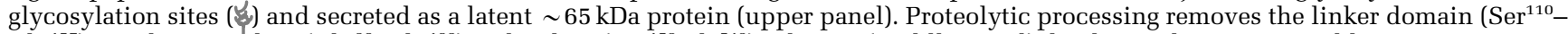
$\mathrm{Gln}^{157}$ ), resulting in $8 \mathrm{kDa}\left(\mathrm{Gln}^{36}-\mathrm{Glu}^{109}\right)$ and $50 \mathrm{kDa}\left(\mathrm{Lys}^{158}-\mathrm{Ile}^{543}\right)$ subunits (middle panel) that heterodimerize to yield an active enzyme. (b) Antibody specificity. Total cell lysate of heparanase-transfected 293 cells was subjected to immunoblotting applying antibody 1453 (left panel), antibody 733 (right panel) and antibody 810 (middle panel). Note that antibodies 733 and 810 preferentially recognize the 50 and $8 \mathrm{kDa}$ heparanase subunits, respectively, vs the latent $65 \mathrm{kDa}$ protein.

\section{Statistical Analysis}

Statistical analysis was performed using SPSS 11.5 software. Kruskal-Wallis test was applied to define differences of inflammation severity and heparanase staining intensity between the four groups included in the study. Mann-Whitney test was performed to check the above-mentioned differences between pair of groups, and Spearman's correlation was employed to define correlation between inflammation severity and the intensity of heparanase staining.

\section{Results}

\section{Patient Clinical Characteristics}

We utilized immunohistochemistry analysis to study heparanase expression in specimens collected from patients with acute or chronic inflammatory conditions. Patients clinical and sociodemographic data are summarized in Table 1. Infectious colitis pathogens included Salmonella (three patients), Campylobacter (three patients), Hymenolepis nana (one patient), and Clostridium difficile (one patient). Inflammatory activity score was compared between 17 surgical specimens of normal colonic tissue, 17 specimens of ulcerative colitis (13 surgical, four endoscopic), 17 specimens of Crohn's disease, and eight specimens of acute infectious colitis. Inflammation activity score was significantly higher in ulcerative colitis and infectious colitis biopsies compared with biopsies of Crohn's disease (Table 1; $P=0.008)$.

\section{Heparanase Immunostaining}

We have developed a polyclonal antibody (733) that preferentially recognizes the $50 \mathrm{kDa}$ heparanase subunit over the latent $65 \mathrm{kDa}$ pro-enzyme (Figure 1b). Thus, immunostaining of archival material can be correlated with heparanase enzymatic activity. We utilized antibody 733 to examine heparanase expression in specimens collected from patients with acute (infectious colitis) and chronic (inflammatory bowel disease) inflammation. No staining of heparanase was detected in normal colon tissue (Table 2, Figure 2a and b), in agreement with previous findings. ${ }^{15,17}$ Similarly, no detectable heparanase staining was evident in infectious colitis specimens (Table 2, Figure 2d and e). In contrast, heparanase staining was markedly increased in Crohn's disease (Table 2, Figure 2g and h) and ulcerative colitis (Table 2, Figure $2 \mathrm{j}$ and $\mathrm{k}$ ) specimens. Surprisingly, only weak heparanase staining was evident in immune cells (Figure 2), whereas prominent staining was detected in epithelial cells of the inflamed colon (Figure $2 \mathrm{~g}-\mathrm{l}$ ). In all positively stained cases, more than $50 \%$ of the epithelial cells reacted with the anti-heparanase antibody. The strongest glandular and surface epithelium (Figure 2j) staining was observed in ulcerative colitis and Crohn's disease patients (scored as $1.7 \pm 0.66$ and $1.4 \pm 0.75$, respectively, Table 2) compared to scoring of $0.3 \pm 0.4$ and $0.44 \pm 0.42$ in normal colonic and infectious colitis specimens, respectively, differences that are statistically highly significant $(P=0.008)$. Moreover, the intensity of heparanase 
Table 1 Patient characteristics and inflammation activity

\begin{tabular}{|c|c|c|c|}
\hline & Ulcerative colitis $(\mathrm{n}=17)$ & Crohn's disease $(\mathrm{n}=17)$ & Infectious colitis $(\mathrm{n}=8)$ \\
\hline Age at diagnosis (years, mean \pm s.d.) & $32.9 \pm 19.7$ & $27.4 \pm 15.9$ & NA \\
\hline Males (\%) & $13(\overline{76})$ & $9(53)$ & $6(75)$ \\
\hline $\begin{array}{l}\text { Disease duration before surgery/endoscopy } \\
\text { (years, mean } \pm \text { s.d.) }\end{array}$ & $6.6 \pm 8.5$ & $6.1 \pm 6.6$ & 2.3 weeks \\
\hline \multirow[t]{5}{*}{ Indication for surgery } & Intractable disease: $12 / 17$ & Intractable disease: $4 / 17$ & \multirow[t]{5}{*}{ NA } \\
\hline & IBD surveilance: $2 / 17$ & Bowel obstruction: 5/17 & \\
\hline & IBD exacerbation: $2 / 17$ & Severe dysplasia: 1/17 & \\
\hline & Bowel obstruction: 1/7 & Disease penetration: 6/17 & \\
\hline & & Unknown: 1/17 & \\
\hline \multirow[t]{4}{*}{ Type of surgery } & Total proctocolectomy: 13/17 & Rt hemicolectomy: 12/17 & \multirow[t]{4}{*}{ NA } \\
\hline & Colonoscopy: 4/17 & Lt. hemicolectomy: 2/17 & \\
\hline & & Total colcetomy: 2/17 & \\
\hline & & Sigmoidectomy: 1/17 & \\
\hline \multirow[t]{3}{*}{ Disease extent } & Proctitis: $0 / 17$ & Terminal ileum: 2/17 & \multirow[t]{3}{*}{ NA } \\
\hline & Left-sided colitis: $4 / 17$ & Ileo-colic: $11 / 17$ & \\
\hline & Pancolitis: 13/17 & Colon-only: 4/17 & \\
\hline \multirow[t]{3}{*}{ Disease behavior } & NA & Inflammatory: $4 / 17$ & \multirow[t]{3}{*}{ NA } \\
\hline & & Penetrating: $7 / 17$ & \\
\hline & & Stricturing: 6/17 & \\
\hline Inflammation activity score (mean \pm s.d.) & $1.88 \pm 0.82$ & $0.74 \pm 0.69$ & $1.62 \pm 0.52$ \\
\hline
\end{tabular}

Table 2 Heparanase staining intensity in normal colonic vs colitis biopsies

\begin{tabular}{lcc}
\hline & $\begin{array}{c}\text { Heparanase } 733 \text { staining } \\
\text { intensity in colonic } \\
\text { epithelial cells } \\
\text { (mean } \pm \text { s.d.) }\end{array}$ & $\begin{array}{c}\text { Proportion of } \\
\text { positively } \\
\text { stained } \\
\text { specimens }\end{array}$ \\
\hline Normal colon & $0.32 \pm 0.43$ & $4^{\mathrm{c} / 17}$ \\
Ulcerative colitis & $1.74 \pm 0.66^{\mathrm{a}, \mathrm{b}}$ & $17 / 17$ \\
Crohn's disease & $1.41 \pm 0.75^{\mathrm{a}, \mathrm{b}}$ & $15 / 17$ \\
Infectious colitis & $0.44 \pm 0.42$ & $2^{\mathrm{c} / 8}$ \\
\hline
\end{tabular}

${ }^{\mathrm{a}}$ Compared to normal colon $P=0.001$.

${ }^{\mathrm{b}}$ Compared to infectious colitis $P=0.008$.

${ }^{\mathrm{C}}$ Weak (+1) staining was observed in these cases.

staining by the inflamed epithelium correlated with inflammation activity in ulcerative colitis and Crohn's disease patients (Spearman's correlation index $\left.r_{\mathrm{s}}=0.404, P=0.018\right)$. In ulcerative colitis specimens, similar heparanase staining was noted for endoscopic (4/17) and full-thickness (13/17) biopsies. No heparanase staining was observed in the muscularis and serosa layers, suggesting for epithelial-specific reactivity. In the inflamed tissue, heparanase staining was mainly confined to a supranuclear aspect of the colon epithelia (Figure $2 \mathrm{~h}$ and $\mathrm{k}$ ) in a pattern that somewhat resembled vesicle-like structures. These vesicles have been previously characterized as endocytic endosomes and lysosomes in cells maintained in vitro, ${ }^{20,22,23}$ as well as in tumor xenografts. ${ }^{20}$ In order to further substantiate this unexpected staining pattern, biopsies were similarly stained with anti-heparanase 810 antibody. This antibody is directed against a peptide mapped at the C-terminus of the $8 \mathrm{kDa}$ subunit that com- prises, together with the $50 \mathrm{kDa}$ subunit, the active heparanase heterodimer enzyme (Figure 1a). ${ }^{21,24}$ Moreover, antibody 810 preferentially recognizes the $8 \mathrm{kDa}$ subunit $v s$ the $65 \mathrm{kDa}$ latent enzyme (Figure $1 \mathrm{~b})^{21}$ and is thus expected to yield staining pattern similar to antibody 733. Indeed, staining with antibody 810 resembled the staining with antibody 733 in terms of specificity, intensity and localization. Thus, no detectable heparanase staining was observed in normal (Figure 2c) and infectious colitis (Figure 2f) specimens. Heparanase was readily detected by antibody 810 in the glandular epithelium of Crohn's disease (Figure 2i) and ulcerative colitis (Figure 2l) specimens, assuming typical supra-nuclear localization (Figure 2i and 1). Staining pattern (intensity and localization) with antibody 810 correlated with antibody 733 staining in 14 out of 16 IBD specimens examined (eight Crohn's disease and eight ulcerative colitis), supporting the notion that the colonic epithelium is a major source of heparanase under chronic, but not acute, inflammatory conditions.

\section{Discussion}

The etiology of inflammatory bowel disease, including Crohn's disease and ulcerative colitis, remains unknown and is likely multifactorial..$^{25}$ Clearly, the immune system plays a key role in either initiating or maintaining the disease state, largely owing to imbalance between pro-, and anti-inflammatory cytokines. ${ }^{18}$ The intestinal epithelium plays an active role in the maintenance of mucosal homeostasis and is considered to be part of the innate 

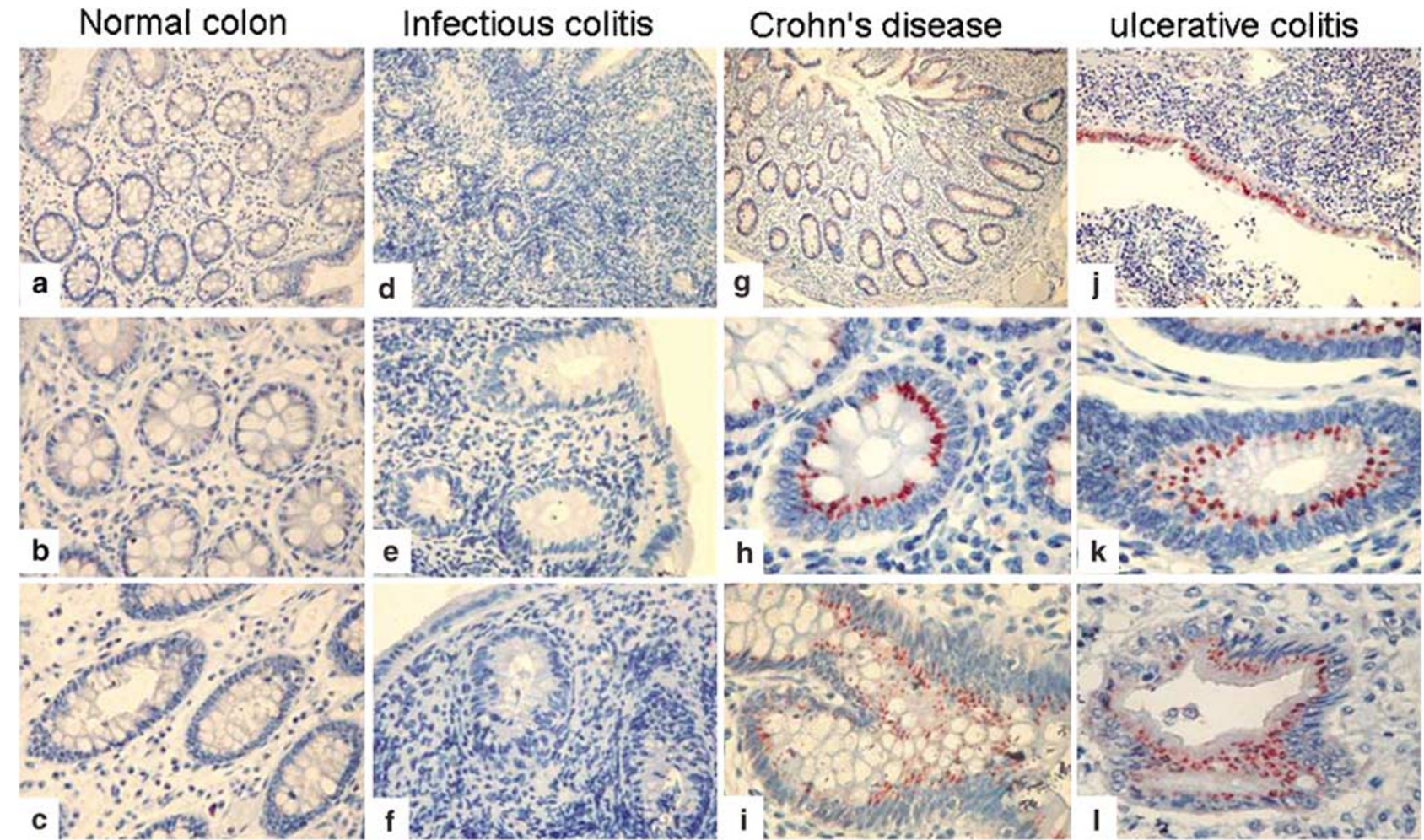

Figure 2 Immunohistochemical staining of heparanase in inflammatory bowel disease specimens. Formalin-fixed, paraffin-embedded $5 \mu \mathrm{m}$ sections of normal (a-c), infectious colitis $(\mathbf{d}-\mathbf{f})$, Crohn's disease (g-i) and ulcerative colitis (j-l) specimens were subjected to immunostaining of heparanase, applying anti-heparanase 733 (upper and middle panels) and 810 (lower panel) antibodies, as described under 'Materials and methods'. Shown are representative photomicrographs. Original magnifications: (a), (d), (g), (j), (k) × 50; (b), (c), (e), (f), (h), (i), (l) $\times 200$. No detectable staining was observed with pre-immune serum, or when the primary antibody was omitted.

immunity system. Epithelial cells can act as antigenpresenting cells and, moreover, are avid producers of chemokines and cytokines considered central to the pathogenesis of inflammatory bowel disease such as tumor necrosis factor (TNF)- $\alpha$, interleukin1 and interleukin-6. ${ }^{26}$ Aberrant secretion of these pro-inflammatory mediators by the epithelium is an integral part of the dysregulated immune response that initiates or perpetuates intestinal inflammation. ${ }^{26}$ The results presented here clearly suggest that colonic glandular and surface epithelial cells are also avid producers of heparanase. Among the few cell types that express heparanase under normal physiological conditions are placental cytothrophoblasts, skin keratinocytes and blood borne cells such as lymphocytes, neutrophils, macrophages and platelets. ${ }^{2,3}$ Heparanase was localized to tertiary granules of neutrophils ${ }^{6,27}$ and mast cells, ${ }^{28}$ and its release by degranulation has been implicated in diapedesis of a number of immune cells, including neutrophils, macrophages, mast cells, dendritic cells and lymphocytes, ${ }^{13,29,30}$ thus strongly implying heparanase as a pro-inflammatory mediator. Surprisingly, however, only a weak heparanase staining was observed in immune cells of IBD specimens (Figure 2). In contrast, the inflamed colonic epithelium exhibited strong reactivity with the anti-heparanase antibodies employed (Table 2, Figure 2), suggesting that colonic epithelium rather than immune cells is the major heparanase producer under chronic inflammatory conditions. We have recently reported that heparanase expression is induced in a delayedtype hypersensitivity inflammatory model in mice. ${ }^{31}$ In the delayed-type hypersensitivity model, the vascular endothelium emerged as the major cellular source of heparanase, and pro-inflammatory mediators such as TNF- $\alpha$ and IFN- $\gamma$ were able to induce heparanase expression by cultured endothelial cells. ${ }^{31}$ It is likely that elevated heparanase levels produced locally by endothelial cells enhance the remodeling of the vascular basement membrane, allowing extravasation of immune cells. ${ }^{31}$ It therefore appears that vascular-, and immune cellsderived heparanase, contributes to the early phases of an inflamed condition, ${ }^{31}$ whereas prolonged exposure to pro-inflammatory cytokines results in heparanase induction by the colonic epithelium, contributing to the sustained, chronic nature of the disease. Interestingly, un-fractionated as well as low molecular weight heparin (enoxaparin), which exhibit a strong inhibitory activity towards heparanase, ${ }^{32-35}$ have proven efficacious in ulcerative colitis and Crohn's disease patients, ${ }^{36-38}$ suggesting that heparanase is actively involved in these pathologies and thus may be considered as a target for the development of anti-inflammatory therapies. 


\section{Acknowledgements}

This work was supported by grants from the Israel Science Foundation (Grant 532/02); National Cancer Institute, NIH (Grant RO1-CA106456); the Israel Cancer Research Fund; and the Rappaport Family Institute Fund.

\section{References}

1 Dempsey LA, Brunn GJ, Platt JL. Heparanase, a potential regulator of cell-matrix interactions. Trends Biochem Sci 2000;25:349-351.

2 Parish CR, Freeman C, Hulett MD. Heparanase: a key enzyme involved in cell invasion. Biochim Biophys Acta 2001;1471:M99-M108.

3 Vlodavsky I, Friedmann Y. Molecular properties and involvement of heparanase in cancer metastasis and angiogenesis. J Clin Invest 2001;108:341-347.

4 Bernfield M, Gotte M, Park PW, et al. Functions of cell surface heparan sulfate proteoglycans. Annu Rev Biochem 1999;68:729-777.

5 Kjellen L, Lindahl U. Proteoglycans: structures and interactions. Annu Rev Biochem 1991;60:443-475.

6 Matzner Y, Bar-Ner M, Yahalom J, et al. Degradation of heparan sulfate in the subendothelial extracellular matrix by a readily released heparanase from human neutrophils. Possible role in invasion through basement membranes. J Clin Invest 1985;76:1306-1313.

7 Nakajima M, Irimura T, DiFerrante D, et al. Heparan sulfate degradation: relation to tumor invasion and metastatic properties of mouse B 16 melanoma sublines. Science (Washington, DC) 1983;220:611-613.

8 Nakajima M, Irimura T, Di Ferrante N, et al. Metastatic melanoma cell heparanase. Characterization of heparan sulfate degradation fragments produced by B16 melanoma endoglucuronidase. J Biol Chem 1984;259: 2283-2290.

9 Vlodavsky I, Fuks Z, Bar-Ner M, et al. Lymphoma cells mediated degradation of sulfated proteoglycans in the subendothelial extracellular matrix: relation to tumor cell metastasis. Cancer Res 1983;43:2704-2711.

10 Edovitsky E, Elkin M, Zcharia E, et al. Heparanase gene silencing, tumor invasiveness, angiogenesis, and metastasis. J Natl Cancer Inst 2004;96:1219-1230.

11 Lider O, Baharav E, Mekori YA, et al. Suppression of experimental autoimmune diseases and prolongation of allograft survival by treatment of animals with low doses of heparins. J Clin Invest 1989;83:752-756.

12 Naparstek Y, Cohen IR, Fuks Z, et al. Activated T lymphocytes produce a matrix-degrading heparan sulphate endoglycosidase. Nature 1984;310:241-244.

13 Vlodavsky I, Eldor A, Haimovitz-Friedman A, et al. Expression of heparanase by platelets and circulating cells of the immune system: possible involvement in diapedesis and extravasation. Invas Metast 1992;12: 112-127.

14 Vaday GG, Lider O. Extracellular matrix moieties, cytokines, and enzymes: dynamic effects on immune cell behavior and inflammation. J Leukoc Biol 2000; 67:149-159.

15 Friedmann Y, Vlodavsky I, Aingorn H, et al. Expression of heparanase in normal, dysplastic, and neoplastic human colonic mucosa and stroma. Evidence for its role in colonic tumorigenesis. Am J Pathol 2000;157:1167-1175.

16 Nobuhisa T, Naomoto Y, Ohkawa T, et al. Heparanase expression correlates with malignant potential in human colon cancer. J Cancer Res Clin Oncol 2005; 131:229-237.

17 Doviner V, Maly B, Kaplan V, et al. Spatial and temporal heparanase expression in colon mucosa throughout the adenoma-carcinoma sequence. Mod Pathol 2006;19:878-888.

18 Bouma G, Strober W. The immunological and genetic basis of inflammatory bowel disease. Nat Rev Immunol 2003;3:521-533.

19 Gasche C, Scholmerich J, Brynskov J, et al. A simple classification of Crohn's disease: report of the Working Party for the World Congresses of Gastroenterology, Vienna 1998. Inflamm Bowel Dis 2000;6:8-15.

20 Zetser A, Levy-Adam F, Kaplan V, et al. Processing and activation of latent heparanase occurs in lysosomes. J Cell Sci 2004;117:2249-2258.

21 Levy-Adam F, Miao HQ, Heinrikson RL. Heterodimer formation is essential for heparanase enzymatic activity. Biochem Biophys Res Commun 2003;308:885-891.

22 Goldshmidt O, Nadav L, Aingorn H, et al. Human heparanase is localized within lysosomes in a stable form. Exp Cell Res 2002;281:50-62.

23 Nadav L, Eldor A, Yacoby-Zeevi O, et al. Activation, processing and trafficking of extracellular heparanase by primary human fibroblasts. J Cell Sci 2002;115: 2179-2187.

24 McKenzie E, Young K, Hircock M, et al. Biochemical characterization of the active heterodimer form of human heparanase (Hpa1) protein expressed in insect cells. Biochem J 2003;373:423-435.

25 Hanauer SB. Inflammatory bowel disease: epidemiology, pathogenesis, and therapeutic opportunities. Inflamm Bowel Dis 2006;12(Suppl 1):S3-S9.

26 Bamias G, Nyce MR, De La Rue SA, et al. New concepts in the pathophysiology of inflammatory bowel disease. Ann Intern Med 2005;143:895-904.

27 Mollinedo F, Nakajima M, Llorens A, et al. Major co-localization of the extracellular-matrix degradative enzymes heparanase and gelatinase in tertiary granules of human neutrophils. Biochem J 1997;327: 917-923.

28 Bashkin $\mathrm{P}$, Doctrow S, Klagsbrun $\mathrm{M}$, et al. Basic fibroblast growth factor binds to subendothelial extracellular matrix and is released by heparitinase and heparin-like molecules. Biochemistry 1989;28: 1737-1743.

29 Bartlett MR, Underwood PA, Parish CR. Comparative analysis of the ability of leucocytes, endothelial cells and platelets to degrade the subendothelial basement membrane: evidence for cytokine dependence and detection of a novel sulfatase. Immunol Cell Biol 1995;73:113-124.

30 Parish CR, Hindmarsh EJ, Bartlett MR, et al. Treatment of central nervous system inflammation with inhibitors of basement membrane degradation. Immunol Cell Biol 1998;76:104-113.

31 Edovitsky E, Lerner I, Zcharia E, et al. Role of endothelial heparanase in delayed-type hypersensitivity. Blood 2006;107:3609-3616.

32 Bar-Ner M, Eldor A, Wasserman L, et al. Inhibition of heparanase-mediated degradation of extracellular matrix heparan sulfate by non-anticoagulant heparin species. Blood 1987;70:551-557. 
33 Vlodavsky I, Mohsen M, Lider O, et al. Inhibition of tumor metastasis by heparanase inhibiting species of heparin. Invas Metast 1994;14:290-302.

34 Miao HQ, Elkin M, Aingorn E, et al. Inhibition of heparanase activity and tumor metastasis by laminarin sulfate and synthetic phosphorothioate oligodeoxynucleotides. Int J Cancer 1999;83:424-431.

35 Ferro V, Hammond E, Fairweather JK. The development of inhibitors of heparanase, a key enzyme involved in tumour metastasis, angiogenesis and inflammation. Mini Rev Med Chem 2004;4: 693-702.
36 Dotan I, Hallak A, Arber N, et al. Low-dose lowmolecular weight heparin (enoxaparin) is effective as adjuvant treatment in active ulcerative colitis: an open trial. Dig Dis Sci 2001;46:2239-2244.

37 Folwaczny C, Wiebecke B, Loeschke K. Unfractioned heparin in the therapy of patients with highly active inflammatory bowel disease. Am J Gastroenterol 1999; 94:1551-1555.

38 Prajapati DN, Newcomer JR, Emmons J, et al. Successful treatment of an acute flare of steroid-resistant Crohn's colitis during pregnancy with unfractionated heparin. Inflamm Bowel Dis 2002;8:192-195. 